Pacific Journal of Mathematic 


\title{
BOUNDARY VALUE PROBLEMS WITH INTERIOR POINT BOUNDARY CONDITIONS ${ }^{1}$
}

\author{
AllaN M. KRALL
}

Recently Neuberger, Zettl and Loud have revived interest in self-adjoint boundary value problems with interior point boundary conditions. All three have derived their results from rather extensive study of the Green's function associated with the nonhomogeneous problem. They require $G(x, \xi)=$ $G(\xi, x)^{*}$.

Rather than approach the problem via the Green's function, this article considers the problem as that of a differential operator in a Hilbert space, derives the adjoint operator, whose domain specifies the adjoint boundary conditions, and then produces necessary and sufficient conditions for selfadjointness.

To do this we employ a variation of the fundamental lemma of the calculus of variations in the Hilbert space setting, and we note our method is applicable even when the Green's function fails to exist.

For convenience we only consider a first order vector equation, although our results are easily extended to $n$-th order vector systems. Finally, our method is extendable to systems whose boundary conditions are applied at an infinite set of points. We hope to pursue this line in a future paper.

1. The problem and its adjoint. Let us consider an interval $[a, b]$ which is subdivided into $m$ subintervals by

$$
a_{1}, a_{2}, \cdots, a_{m-1}\left(a=a_{0}<a_{1}<\cdots<a_{m-1}<a_{m}=b\right) .
$$

We denote by $H$ the Hilbert space of $n \times 1$ vectors

$$
X=\left(x_{1}, x_{2}, \cdots, x_{n}\right)^{t}, \quad Y=\left(y_{1}, y_{2}, \cdots, y_{n}\right)^{t},
$$

defined on $[a, b]$ whose components are in $L^{2}(a, b)$ and whose inner product is given by

$$
(X, Y)=\int_{a}^{b} Y^{*} X d x=\sum_{i=1}^{\infty} \int_{a}^{b} x_{i} \bar{y}_{i} d x .
$$

Let us consider boundary operators of the form

$$
M_{i} Y=\sum_{i=0}^{m}\left[A_{i j} Y\left(a_{j}+\right)+B_{i j} Y\left(a_{j}-\right)\right],
$$

$i=1, \cdots, k$, where $A_{i m}=0, B_{i 0}=0$, and $Y\left(a_{j} \pm\right)$ indicates the limit of 
$Y(x)$ as $x$ approaches $a_{j}$ from above or below. We assume that these boundary operators are linearly independent. Thus $k \leqq 2 n m$. Note, however, $k$ does not have to equal $n m$.

We let $A_{1}$ and $A_{0}$ be continuous $n \times n$ matrices, and in addition assume that $A_{1}^{\prime}(x)$ exists and is continuous. We denote by $D$ the set of all $n \times 1$ vectors $Y$ satisfying

(1) $Y$ is in $H$.

(2) $Y$ is absolutely continuous in each subinterval $\left[a_{j}, a_{j+1}\right], j=$ $0,1, \cdots, m-1$, of $[a, b]$.

(3) $M_{i} Y=0, i=1, \cdots, k$.

(4) $A_{1} Y^{\prime}+A_{0} Y$ is in $H$.

We define a differential operator $L$ by letting $L Y=A_{1} Y^{\prime}+A_{0} Y$ for all $Y$ in $D$.

It is evident that $D$ is dense in $H$, and therefore $L$ has a welldefined adjoint operator $L^{*}$ associated with it.

THeOREM 1. If $Z$ is in the domain of $L^{*}$, then $Z$ is absolutely continuous in each subinterval $\left[a_{j}, a_{j+1}\right], j=0,1, \cdots, m-1$ of $[a, b]$. $L^{*} Z=-\left(A_{1}^{*} Z\right)^{\prime}+A_{0}^{*} Z$ in each subinterval

$$
\left(a_{j}, a_{j+1}\right), j=0,1, \cdots, m-1
$$

of $[a, b]$.

Proof. Let $H_{0}$ denote the subspace of $D$ whose elements vanish at $a, a_{1}, \cdots, a_{m-1}, b . H_{0}$ is also dense in $H$.

If $Y$ is in $H_{0}$, then

$$
\int_{a}^{b}\left(L^{*} Z\right)^{*} Y d x=\int_{a}^{b} Z^{*}\left[A_{1} Y^{\prime}+A_{0} Y\right] d x .
$$

Thus

$$
\int_{a}^{b} Z^{*} A_{1} Y^{\prime} d x=\int_{a}^{b}\left[L^{*} Z-A_{0}^{*} Z\right]^{*} Y d x .
$$

Since $Y$ vanishes at $a, a_{1}, \cdots, a_{m-1}, b$, integrating by parts,

$$
\int_{a}^{b}\left(A_{1}^{*} Z\right)^{*} Y^{\prime} d x=-\int_{a}^{b}\left\{\int_{a}^{x}\left[L^{*} Z-A_{0}^{*} Z\right] d t\right\}^{*} Y^{\prime} d x \text {. }
$$

So

$$
\int_{a}^{b}\left\{A_{1}^{*} Z+\int_{a}^{x}\left[L^{*} Z-A_{0}^{*} Z\right] d t\right\}^{*} Y^{\prime} d x=0 .
$$

We must now find those elements $J$ such that $\int_{a}^{b} J^{*} Y^{\prime} d x=0$. It is easily seen that $\int_{a}^{b} K^{*} Y d x=0$ if and only if 


$$
\int_{a}^{b}\left[\int_{a}^{x} K^{*} d t\right] Y^{\prime} d x=0
$$

Since $Y$ is in $H_{0}$, which is dense $H, Y^{\prime}$ is only orthogonal to elements which are constant on each subinterval $\left(a_{j}, a_{j+1}\right), j=0,1, \cdots, m-1$. Thus

$$
A_{1}^{*} Z+\int_{a}^{x}\left[L^{*} Z-A_{0}^{*} Z\right] d t=C(Z),
$$

where $C(Z)$ is constant on each subinterval $\left(a_{j}, a_{j+1}\right), j=0,1, \cdots, m-1$. If $x \neq a_{j}$ for some $j$, we may differentiate, and

$$
L^{*} Z=-\left(A_{1}^{*} Z\right)^{\prime}+A_{0}^{*} Z \text {. }
$$

THEOREM 2. If $Z$ is in the domain of $L^{*}$, then $Z$ satisfies the following equations.

$$
\begin{aligned}
& A_{1}^{*}\left(\alpha_{j}-\right) Z\left(a_{j}-\right)-\sum_{i=1}^{k} B_{i j}^{*} \phi_{i}(Z)=0, \\
& \quad-A_{1}^{*}\left(a_{j-1}+\right) Z\left(a_{j-1}\right)-\sum_{i=1}^{k} A_{i j-1}^{*} \phi_{i}(Z)=0,
\end{aligned}
$$

where $j=1,2, \cdots, m$, and $\phi_{i}(Z)$ are functionals which depend upon $Z$.

Proof. Let $Y$ be in the domain of $L$ and $Z$ be in the domain of $L^{*}$. Then

$$
\begin{aligned}
(L Y, Z)-\left(Y, L^{*} Z\right) & =\int_{a}^{b}\left[Z^{*}(L Y)-\left(L^{*} Z\right)^{*} Y\right] d x \\
& =\int_{a}^{b}\left(Z^{*} A_{1} Y\right)^{\prime} d x \\
& =\sum_{j=1}^{m} \int_{a_{j-1}}^{a_{j}}\left(Z^{*} A_{1} Y\right)^{\prime} d x \\
& =\left.\sum_{j=1}^{m}\left(Z^{*} A_{1} Y\right)\right|_{a_{j-1}} ^{a_{j}}=0 .
\end{aligned}
$$

Let $\phi_{i}^{*}(Z)$ be arbitrary parameters, $i=1, \cdots, k$. Then, since $M_{i} Y=0, i=1, \cdots, k$,

$$
0=\left.\sum_{j=1}^{m}\left(Z^{*} A_{1} Y\right)\right|_{a_{j-1}} ^{a_{j}}-\sum_{i=1}^{k} \phi_{i}^{*}(Z) \sum_{j=0}^{m}\left[A_{i j} Y\left(a_{j}+\right)+B_{i j} Y\left(a_{j}-\right)\right] .
$$

Collecting like terms,

$$
\begin{aligned}
0= & \sum_{j=1}^{m}\left[Z\left(a_{j}-\right)^{*} A_{1}\left(a_{j}-\right)-\sum_{i=1}^{k} \phi_{i}^{*}(Z) B_{i j}\right] Y\left(a_{j}-\right) \\
& +\sum_{j=0}^{m-1}\left[-Z\left(a_{j}+\right)^{*} A_{1}\left(a_{j}+\right)-\sum_{i=1}^{k} \phi_{i}^{*}(Z) A_{i j}\right] Y\left(a_{j}+\right) .
\end{aligned}
$$


Since $Y\left(a_{j}+\right)$ and $Y\left(a_{j}-\right)$ may be arbitrary, the result follows.

The parameters $\phi_{i}(Z)$ seem somewhat artificial in this setting. However, if the boundary conditions also involve an integral, they enter in a very natural way, not only into the adjoint boundary conditions, but also into the form of the adjoint operator.

2. Reduction to an end point problem. The results of this section are very similar to a procedure of Mansfield's [2]. Mansfield, however, parameterized each subinterval $\left[a_{j} a_{j+1}\right], j=0, \cdots, m-1$. This is unnecessary.

We make the following definitions. Let $I_{j}=\left[a_{j-1}, a_{j}\right], j=1, \cdots, m$. Let $\mathscr{Y}$ denote the $n m \times 1$ vector $\mathscr{Y}=\left(Y\left(x_{1}\right), Y\left(x_{2}\right), \cdots, Y\left(x_{m}\right)\right)^{t}$ where $x_{j}$ is in $I_{j}$,

$$
\begin{aligned}
& \mathscr{A}_{1}=\left(\begin{array}{cccc}
A_{1}\left(x_{1}\right) & 0 & \cdots & 0 \\
0 & A_{1}\left(x_{2}\right) & \cdots & 0 \\
\vdots & \vdots & & \vdots \\
0 & 0 & \cdots & A_{1}\left(x_{m}\right)
\end{array}\right), \\
& \mathscr{A}_{0}=\left(\begin{array}{cccc}
A_{0}\left(x_{1}\right) & 0 & \cdots & 0 \\
0 & A_{0}\left(x_{2}\right) & \cdots & 0 \\
\vdots & \vdots & & \vdots \\
0 & 0 & \cdots & A_{0}\left(x_{m}\right)
\end{array}\right),
\end{aligned}
$$

$\mathscr{A}=\left(A_{i j-1}\right), \mathscr{B}=\left(B_{i j}\right)$. Let $A$ consist of the $m$-tuple

$$
\left(a_{0}+, a_{1}+, \cdots, a_{m-1}+\right) \text {, }
$$

$B$ the $m$-tuple $\left(a_{1}-, a_{2}-, \cdots, a_{m}-\right)$. By $\mathscr{Y}(A)$ we mean the $n m \times 1$ vector $\left(Y\left(a_{0}+\right), Y\left(a_{1}+\right), \cdots, Y\left(a_{m-1}+\right)^{t}\right.$ with a similar expression for $\mathscr{Y}(B)$.

$H$ is exactly equivalent to the Hilbert space $\mathscr{\mathscr { C }}$ of $n m \times 1$ vectors $\mathscr{Y}$, where the norm is computed by integrating the first $n$ components over $I_{1}$, the next $n$ over $I_{2}$, etc. In this notation, however $D$ corresponds to the set $\mathscr{D}$ which consists of all $n m \times 1$ matrices $\mathscr{Y}$ satisfying

(1) $\mathscr{Y}$ is in $\mathscr{C}$.

(2) $\mathscr{Y}$ is absolutely continuous in the $m$-tuple interval $[A, B]$.

(3) $\mathscr{A} \mathscr{Y}(A)+\mathscr{B} \mathscr{Y}(B)=0$.

(4) $\mathscr{A}_{1} \mathscr{Y}^{\prime}+\mathscr{A}_{0} \mathscr{Y}$ is in $\mathscr{H}$.

Then $L$ corresponds to the operator $\mathscr{L}$ which is defined by $\mathscr{L} \mathscr{Y}=$ $\mathscr{A}_{1} \mathscr{Y}^{\prime}+\mathscr{A}_{0} \mathscr{Y}$ for all $\mathscr{Y}$ in $\mathscr{D}$.

In this setting our problem has been reduced to one with end point boundary conditions.

If $\mathscr{Z}=\left(Z\left(x_{1}\right), Z\left(x_{2}\right), \cdots, Z\left(x_{m}\right)\right)^{t}$ and 


$$
\Phi(\mathscr{Z})=\left(\phi_{1}(Z), \phi_{2}(Z), \cdots, \phi_{k}(Z)\right)^{t},
$$

the adjoint operator takes the form.

$$
\mathscr{L}^{*} \mathscr{Z}=-\left(\mathscr{L}_{1}^{*} \mathscr{Z}\right)^{\prime}+\mathscr{L}_{0}^{*} \mathscr{Z}
$$

on $[A, B]$. The domain of $\mathscr{L}^{*}$ is determined by the boundary conditions

$$
\begin{aligned}
& \mathscr{A}_{1}^{*}(A) \mathscr{Z}(A)+\mathscr{A}^{*} \Phi(\mathscr{Z})=0, \\
& \mathscr{A}_{1}^{*}(B) \mathscr{Z}(B)-\mathscr{R}^{*} \Phi(\mathscr{Z})=0 .
\end{aligned}
$$

Green's formula takes the form

$$
\begin{aligned}
\int_{A}^{B}\left[\mathscr{Z}^{*}(\mathscr{C} \mathscr{Y})-\right. & \left.\left(\mathscr{L}^{*} \mathscr{Z}\right)^{*} \mathscr{Y}\right] d x=\left.\left(\mathscr{Z}^{*} \mathscr{\mathscr { A }} \mathscr{Y}\right)\right|_{A} ^{B} \\
& =\Phi(\mathscr{\mathscr { C }})^{*}[\mathscr{\mathscr { Y }} \mathscr{Y}(A)+\mathscr{B} \mathscr{Y}(B)] \\
& =0 .
\end{aligned}
$$

On the other hand if $\mathscr{C} \mathscr{Y}(A)+\mathscr{D} \mathscr{Y}(B)$ completes the number of independent boundary forms, then there exist complimentary forms $\tilde{\mathscr{A}} \mathscr{Z}(A)+\tilde{\mathscr{B}} \mathscr{\mathscr { F }}(B)$ and $\tilde{\mathscr{C}} \mathscr{\mathscr { C }}(A)+\tilde{\mathscr{D}} \mathscr{F}(B)$ such that

$$
\begin{aligned}
& \int_{4}^{B}\left[\mathscr{Z} *(\mathscr{L} \mathscr{Y})-\left(\mathscr{L}^{*} \mathscr{Z}\right) \mathscr{Y}\right]^{*} d X \\
& =[\tilde{\mathscr{A}} \mathscr{Z}(A)+\tilde{\mathscr{B}} \mathscr{L}(B)]^{*}[\mathscr{A} \mathscr{Y}(A)+\mathscr{B} \mathscr{Y}(B)] \\
& +[\tilde{\mathscr{C}} \mathscr{Z}(A)+\tilde{\mathscr{D}} \mathscr{\mathscr { L }}(B)]^{*}[\mathscr{\mathscr { C }} \mathscr{Y}(A)+\mathscr{D} \mathscr{Y}(B)] \text {. }
\end{aligned}
$$

The coefficients of these forms satisfy

$$
\left(\begin{array}{cc}
\tilde{\mathscr{A}}^{*} & \tilde{\mathscr{D}}^{*} \\
\tilde{\mathscr{B}}^{*} & \tilde{\mathscr{D}}^{*}
\end{array}\right)\left(\begin{array}{cc}
\mathscr{A} & \mathscr{B} \\
\mathscr{C} & \mathscr{D}
\end{array}\right)=\left(\begin{array}{cc}
-\mathscr{A}_{1}(A) & 0 \\
0 & \mathscr{A}_{1}(B)
\end{array}\right)
$$

which yields the equivalent boundary condition $\tilde{\mathscr{C}} \mathscr{F}(A)+\tilde{\mathscr{D}} \mathscr{\mathscr { Z }}(B)=0$, and the formula $\Phi(\mathscr{Z})=\tilde{\mathscr{A}} \mathscr{Z}(A)+\tilde{\mathscr{B}} \mathscr{\mathscr { Z }}(B)$. The following theorem follows in a manner similar to that of Reid [4].

THEOREM 3. $\mathscr{P}$ is self-adjoint in $\mathscr{P}$ if and only if

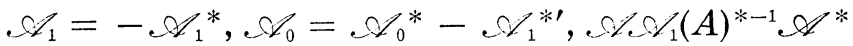

$$
\begin{aligned}
& =\mathscr{B} \cdot \mathscr{L}_{1}(B)^{*-1} \cdot \mathscr{B}^{*} \text {. }
\end{aligned}
$$

The last result may also be found by substituting the parametric adjoint boundary conditions into those for $\mathscr{L}$.

If the number of boundary conditions $k$ in the original problem is equal to $n m$, and if the homogeneous problem $(\mathscr{L}-\lambda) \mathscr{Y}=0$ has only the trivial solution in $\mathscr{C}$, then the nonhomogeneous problem $(\mathscr{L}-\lambda) \mathscr{Y}=\mathscr{F}$ has a unique solution, which is generated by an in- 
tegral equation.

$$
\mathscr{Y}(X)=\int_{A}^{B} \mathscr{D}(X, \Xi) \mathscr{F}(\Xi) d \Xi
$$

The Green's function $\mathscr{D}(X, \Xi)$ has the form

$$
\left(\begin{array}{cccc}
G\left(x_{1} \times x_{1}\right) & G\left(x_{1} \times x_{2}\right) & \cdots & G\left(x_{1} \times x_{m}\right) \\
G\left(x_{2} \times x_{1}\right) & G\left(x_{2} \times x_{2}\right) & \cdots & G\left(x_{2} \times x_{m}\right) \\
\vdots & \vdots & \vdots \\
G\left(x_{m} \times x_{1}\right) & G\left(x_{m} \times x_{2}\right) & \cdots & G\left(x_{m} \times x_{m}\right)
\end{array}\right) .
$$

As a function of $X$ it formally satisfies $(\mathscr{L}-\lambda) \mathscr{Y}=0$ and the boundary conditions defining $\mathscr{D}$. As a function of $\Xi, \mathscr{D}(X, \Xi)^{*}$ formally satisfies the adjoint equation $\left(\mathscr{L}^{*}-\lambda\right) \mathscr{Z}=0$ and the adjoint boundary conditions. If $\mathscr{L}$ is self-adjoint in $\mathscr{H}, \mathscr{D}(X, \Xi)$ automatically exhibits the usual symmetric properties associated with self-adjoint boundary value problems which were illustrated by Loud [1].

We finally remark that these results can be extended to higher order systems with the standard modifications. In the self-adjoint situations the usual eigenfunction expansions are valid. In the nonself-adjoint situations expansions similar to Birkhoff's are possible.

\section{REFERENCES}

1. W.S. Loud, Self-adjoint multipoint boundary value problems, Pacific J. Math. 20 (1968), 303-317.

2. R. Mansfield, Differential systems involving k-point boundary conditions, Cont. Calc. Var. (Chicago 1942), 413-452.

3. J. W. Neuberger, The lack of self-adjointness in three point boundary value problems, Pacific J. Math. 18 (1966), 165-168.

4. W. T. Reid, A new class of two point boundary value problems, Illinois J. Math. 2 (1958), 434-453.

5. A. Zettl, The lack of self-adjointness in three point boundary value problems, Proc. Amer. Math. Soc. 17 (1966), 368-371.

6. - Adjoint and self-adjoint boundary value problems with interface conditions, MRC Tech. Rept. $¥ 827,1967$.

Received June 3, 1968. This reseach was supported in part by NASA Grant NGR 39-009-041.

Pennsyluania State University

University Park, Pennsylvania 



\section{Pacific Journal of Mathematics \\ Vol. 29, No. 1 \\ May, 1969}

Jorge Alvarez de Araya, A Radon-Nikodým theorem for vector and operator

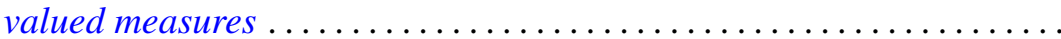

Deane Eugene Arganbright, The power-commutator structure of finite

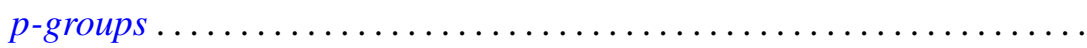

Richard Eugene Barlow, Albert W. Marshall and Frank Proschan, Some

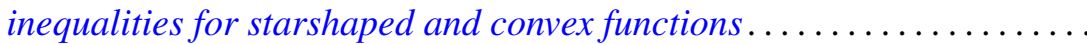

David Clarence Barnes, Some isoperimetric inequalities for the eigenvalues

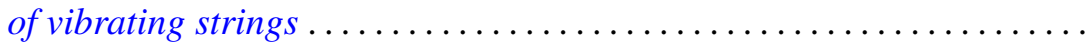

David Hilding Carlson, Critical points on rim-compact spaces ...........

Allan Matlock Weber Carstens, The lattice of pretopologies on an arbitrary

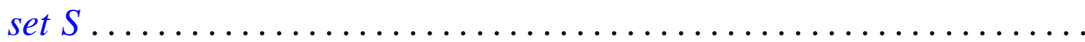

S. K. Chatterjea, A bilateral generating function for the ultraspherical

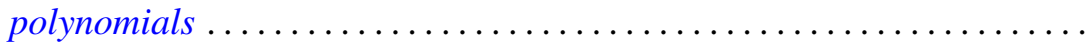

Ronald J. Ensey, Primary Abelian groups modulo finite groups ......... 77

Harley M. Flanders, Relations on minimal hypersurfaces ............ 83

Allen Roy Freedman, On asymptotic density in n-dimensions........... 95

Kent Ralph Fuller, On indecomposable injectives over artinian rings...... 115

George Isaac Glauberman, Normalizers of p-subgroups in finite groups . . . 137

William James Heinzer, On Krull overrings of an affine ring ........... 145

John McCormick Irwin and Takashi Ito, A quasi-decomposable abelian group without proper isomorphic quotient groups and proper isomorphic subgroups.

Allan Morton Krall, Boundary value problems with interior point boundary conditions

John S. Lowndes, Triple series equations involving Laguerre

polynomials

Philip Olin, Indefinability in the arithmetic isolic integers

Ki-Choul Oum, Bounds for the number of deficient values of entire functions whose zeros have angular densities..

R. D. Schafer, Standard algebras ....................

Wolfgang M. Schmidt, Irregularities of distribution. III.

Richard Alfred Tapia, An application of a Newton-like method to the Euler-Lagrange equation 